\title{
DOI: https://doi.org/10.24297/jam.v18i.8543
}

\section{Regional Boundary Asymptotic Gradient Full-Order Observer in Distributed Parabolic Systems}

\author{
Zinah A. Khalid ${ }^{1}$ and Raheam A. Al-Saphory ${ }^{2}$ \\ 1,2Department of Mathematics, College of Education for Pure Sciences, Tikrit University, Tikrit, Iraq. \\ saphory@hotmail.com²zeena_assif@hotmail.com¹
}

\begin{abstract}
The purpose of this paper is to explore the concept of the regional boundary asymptotic gradient full order observer (RBAGFO-observer) in connection with the characterizations of sensors structures. Then, we present various results related to different types of measurements, domains and boundary conditions for distributed parameter systems (DPSs) in parabolic systems problem. The considered approach of this work is derived from Luenberger observer theory which is enable to estimate asymptotically the state gradient of the original system on a sub-region of the domain boundary $\partial \Omega$ in order that the RBAGFO-observability notion to be achieved. We also show that there exists a dynamical system for the considered system is not BAGFO-observer in the usual sense, but it may be regional RBAGFO-observer.
\end{abstract}

Keywords: RBG-strategic sensors, RBAG-detectability, RBAGFO-observers, diffusion system.

Mathematics Subject Classification: 93A30; 93B07; 93B30; 93C2.

\section{Introduction}

There are many situations in modern technology in which it is necessary to estimate the state of a dynamic system using only the measured input and output data of the system [1]. An observer is a dynamic system $\hat{S}$ which to estimates the state of another considered system $\mathrm{S}$ using only the measured input and output letter. If the order of $\hat{S}$ is equal to the order of $S$, the observer is called full-order state observer [2-4].

The asymptotic observer theory explored by Luenberger in [5] for finite-dimensional linear systems and extended infinite-dimensional distributed parameter systems govern by strongly continuous semi-group in Hibert space by Gressang and Lamont as in [6]. The study of this approach via another variable like sensors and actuators developed by El-Jai et al. as in ref.s [2-7] in order to achieve asymptotic observability.

One of the most important approach in system theory is focused on the reconstruction of the state of the system from the knowledge of dynamic system and the output function on a sub-region $\omega$ of a spatial domain $\Omega$ this problem is called regional observability problem has been received much attention as in [8-10].

An extension of this notion has been given in [11-12] to the regional gradient case. The regional asymptotic notion has been introduced and developed by Al-Saphory and El- Jai in [13-14]. Thus, this notion consists in studying the asymptotic behavior of the system in an internal sub-region $\omega$ of a spatial domain $\Omega$.

Thus, the asymptotic regional state reconstruction studied and developed in [15-16] and extended to the regional asymptotic gradient full-order observer (RGFO-observer) which allows estimating the state gradient of the original system.

The purpose of this paper is to study and examine the concept of RBAGFO-observer by using the choice of sensors. The principle reason for considering this case is that, in the first time, the existent of a dynamical system which is observed asymptotically the gradient of the system state on some boundary region $\ulcorner\subset \partial \Omega$ [17]. In second time, it is closer to a real situation, the treatment of water by using a bioreactor where the objective is to observe the concentration of substrate at the boundary output of the bioreactor in order the water regulation is achieved (for example see figure 1). 


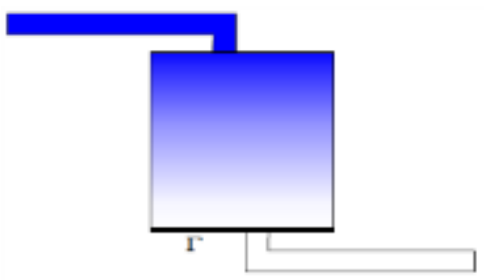

Fig. 1: Observation of substrate concentration at the boundary output of the reactor.

The outline of this paper is organized as follows: Section 2 is devoted to the problem statement and some basic concept related to the regional boundary gradient stability (RBAG-stability), regional boundary asymptotic gradient detectability (RBAG-detectability). Section 3, we focus on RBAGFO-observer so we introduced and characterize the existing of RBAGFO-observer to provide RBAGFO-estimator of gradient state for the original system in terms of sensors structure. In the last section, we have been applied these result to the two (DPSs) for different zone and pointwise sensors case.

\section{Problem Formulation and Preliminaries}

This section present considered system and formulation of the problem with some definitions and characterizations, which is related to the present work.

\subsection{Problem Statement}

Let $\Omega$ be an open bounded subset of $R^{n}$ with boundary $\partial \Omega$ and $\Gamma$ be a region subset of $\partial \Omega$. We denote $Q=$ $\Omega \times] 0, \infty[$ and $\Sigma=\partial \Omega \times] 0, \infty[$. Consider the parabolic system which is described by the following state-space equation

$$
\begin{cases}\frac{\partial x}{\partial t}(\xi, t)=A x(\xi, t)+B u(t) & Q \\ x(\xi, 0)=x_{0}(\mu) & \bar{\Omega} \\ \frac{\partial x}{\partial v}(\eta, t)=0 & \Sigma\end{cases}
$$

augmented with the output function

$$
y(., t)=C x(., t)
$$

(2) - The separable Hilbert spaces are $X, U$ and $\mathcal{O}$ where $X=H^{1}(\bar{\Omega})$ is the state space, $U=L^{2}\left(0, T, R^{p}\right)$ is the control space and $\mathcal{O}=$ $L^{2}\left(0, T, R^{q}\right)$ is the observation space, where $p$ and $q$ are the numbers of actuators and sensors.

- $A=\sum_{i, j=1}^{n} \frac{\partial}{\partial x_{j}}\left(a_{i j} \frac{\partial}{\partial x_{j}}\right)$ with $a_{i j} \in D(\bar{A})$ (the domain of $\bar{A}$ ) is a second-order linear differential operator, which generates a strongly continuous semi-group $\left(S_{A}(t)\right)_{t \geq 0}$ on the state space $X$ and is self-adjoint with compact resolvent [18].

- The operators $B \in L\left(R^{p}, X\right)$ and $C \in L\left(X, R^{q}\right)$ depend on the structure of actuators and sensors as in [19] (figure 2)

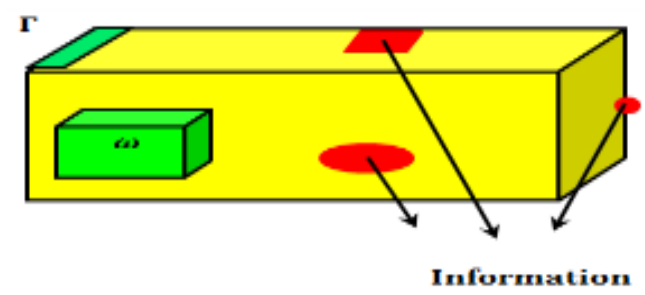

Fig. 2: Mathematical model: domain $\Omega$, region $\Gamma$, and sensors Locations. 
- The mathematical model in figure 2 is more general and complex than the the spatial case of real model in figure 1.

- Under the given assumptions above, the system (1) has a unique solution given by the following form [20-21].

$$
x(\xi, t)=S_{A}(t) x_{0}(\xi)+\int_{0}^{t} S_{A}(t-s) B u(s) d s
$$

- The problem is how to reconstruct a dynamical system may be called estimator for the current state gradient in a given region on $\Gamma$, and to give a sufficient condition for the existence of a RBAGFO-observer.

- The initial state $x_{0}$ and its gradient $\nabla x_{0}$ are supposed to be unknown, the problem concerns the reconstruction of the initial gradient $\nabla x_{0}$ on the region $\Gamma$ of the system domain $\partial \Omega$.

- Now, we consider the operator $K$ given by the form

$$
\begin{aligned}
K: X & \rightarrow \mathcal{O} \\
x & \rightarrow C S_{A}(.) x
\end{aligned}
$$

where $K$ is a bounded linear operator as in $[7,22,24]$. And the adjoint operator $K^{*}$ of $K$ is defined by

$$
\begin{aligned}
K^{*}: \mathcal{O} \rightarrow X, \text { and represented by the form } \\
K^{*} y^{*}=\int_{0}^{t} S_{A}^{*}(s) C^{*} y^{*}(s) d s
\end{aligned}
$$

- The operator $\nabla$ denotes the gradient is given by

$$
\left\{\begin{array}{l}
\nabla: H^{1}(\Omega) \rightarrow\left(H^{1}(\Omega)\right)^{n} \\
x \rightarrow \nabla_{x}=\left(\frac{\partial x}{\partial \xi_{1}}, \ldots, \frac{\partial x}{\partial \xi_{n}}\right)
\end{array}\right.
$$

and, the adjoint of $\nabla$ denotes by $\nabla^{*}$ is given by

$$
\left\{\begin{array}{l}
\nabla^{*}:\left(H^{1}(\Omega)\right)^{n} \rightarrow H^{1}(\Omega) \\
x \rightarrow \nabla_{x}^{*}=v
\end{array}\right.
$$

where $v$ is a solution of the Dirichlet problem

$$
\begin{cases}\Delta_{v}=-\operatorname{div}(x) & \Omega \\ v=0 & \partial \Omega\end{cases}
$$

- The trace operator of order zero is described by [23]

$$
\gamma_{0}: H^{1}(\Omega) \rightarrow H^{1 / 2}(\partial \Omega)
$$

which is a linear, subjective, and continuous [20]. Thus, the extension of the trace operator [23] which is denoted by $\gamma$ defined as

$$
\gamma:\left(H^{1}(\Omega)\right)^{n} \rightarrow\left(H^{1 / 2}(\partial \Omega)\right)^{n}
$$

and the adjoints are respectively given by $\gamma_{0}{ }^{*}, \gamma^{*}$.

- For a region $\Gamma$ of $\partial \Omega$, we define the gradient restriction operator by the form 


$$
\chi_{\Gamma}:\left(H^{1 / 2}(\partial \Omega)\right)^{n} \rightarrow\left(H^{1 / 2}(\Gamma)\right)^{n}
$$

where the adjoint of $\chi_{\Gamma}$ denotes by $\chi_{\Gamma}^{*}$ is defined by

$$
\chi_{\Gamma}^{*}:\left(H^{1 / 2}(\Gamma)\right)^{n} \rightarrow\left(H^{1 / 2}(\partial \Omega)\right)^{n}
$$

- Finally, we denote the operator $H_{\Gamma^{*} G}=\chi_{\Gamma} \gamma \nabla K^{*}$ from $\mathcal{O}$ into $\left(H^{1 / 2}(\Gamma)\right)^{n}$ and the adjoint of this operator given by $H_{\Gamma^{*} G}^{*}=K \nabla^{*} \gamma^{*} \chi_{\Gamma}^{*}$.

- For a sub-region $\Gamma$ of the boundary regional boundary gradient $\Gamma_{R B G}=\Gamma^{*}$.

Now, the problem is how to build an approach which observe (estimates) regional state gradient on a region $\Gamma^{*}$ of the boundary $\partial \Omega \subset \bar{\Omega}$ asymptotically by using a dynamic system (an observer) in full order case only may be called full-order observer in region $\Gamma^{*}$. The important of an observer is that to estimates all the gradient of state variables, regardless of whether some are available for direct measurements or not [1].

\section{2 $\Gamma^{*}$ G-observability and $\Gamma^{*}$ AG-detectability}

This sub section devotes the relation between the concept of RBG-observability and RBAG-detectability on $\Gamma^{*}$. As well known the observability [19-22] and asymptotic observability [1-5, 7, 24] are important concepts to estimate the unknown state of the considered dynamic system from the input and output functions. Thus, These notions are studied and introduced to the DPSs analysis with different characterizations by El-Jai, Zerrik and AlSaphory et al. in many paper for example $[8-18,28-31]$ in connection with strategic sensors.

- The systems (1)-(2) are said to be exactly regionally boundary gradient observable (E$\Gamma^{*} G$-observable) on $\Gamma^{*}$ if

$$
\operatorname{Im} H=\operatorname{Im} \chi_{\Gamma^{*}} \nabla K^{*}=\left(H^{1 / 2}\left(\Gamma^{*}\right)\right)^{n}
$$

- The systems (1)-(2) are said to be weakly regionally boundary gradient observable (W $\Gamma^{*} \mathrm{G}$-observable) on $\Gamma^{*}$ if

$$
\overline{\operatorname{Im} H}=\overline{\operatorname{Im} \chi_{\Gamma^{*}} \nabla K^{*}}=\left(H^{1 / 2}\left(\Gamma^{*}\right)\right)^{n}
$$

It is equivalent to say that the systems (1)-(2) are $W \Gamma^{*} G$-observable if

$$
\operatorname{Ker} H^{*}=\operatorname{kerK} \nabla^{*} \chi_{\Gamma^{*}}=\{0\}
$$

- If the systems (1)-(2) are is $\mathrm{W} \Gamma^{*} \mathrm{G}$-observable, then $x_{0}(\xi, 0)$ is given by

$$
x_{0}=\left(K^{*} K\right)^{-1} K^{*} y=K^{\dagger} y,
$$

where $K^{\dagger}$ is the pseudo-inverse of the operator $K[9-10]$.

- A sensor $(D, f)$ is a regional boundary gradient strategic ( $\Gamma^{*} \mathrm{G}$-strategic) on $\Gamma^{*}$ if the observed system is W $\Gamma^{*} \mathrm{G}$ observable.

- The measurements can be obtained by the use of zone or pointwise sensors, which may be located in $\Omega$ or $\partial \Omega$ [24].

- Then, according to the choice of the parameters $D_{i}$ and $f_{i}$, we have different types of sensors:

- It may be zone, if $D_{i} \subset \bar{\Omega}$ and $f_{i} \in L^{2}\left(D_{i}\right)$. In this case, the operator $C$ is bounded, and the output function (2) may be given by the form 


$$
y(t)=\int_{D_{i}} z(\xi, t) f_{i}(\xi) d \xi
$$

- It may be pointwise, if $D_{i}=\left\{b_{i}\right\}$ with $b_{i} \in \bar{\Omega}$ and $f=\delta\left(.-b_{i}\right)$ where $\delta$ is a Dirac mass concentrated in $b$ [14, $21,28]$. In this case, the operator $C$ is un bounded, and the output function (2) may be given by the form

$$
y(t)=\int_{\Omega} x(\xi, t) \delta_{b_{i}}\left(\xi-b_{i}\right) d \xi
$$

- It may be boundary zone, if $\Gamma_{i} \subset \partial \Omega$ and $f_{i} \in L^{2}\left(\Gamma_{i}\right)$, the output function (2) may be given by the form

$$
y(t)=\int_{\Gamma_{i}} x(\eta, t) f_{i}(\eta) d \eta
$$

Definition 2.1: The semi-group $\left(S_{A}(t)\right)_{t \geq 0}$ is regionally boundary asymptotically gradient stable ( $\Gamma^{*} \mathrm{AG}$-stable) on $\Gamma^{*}$, if and only if for some positive constants $M_{\Gamma^{*}}, \alpha_{\Gamma^{*}}$, we have

$$
\left\|\chi_{\Gamma^{*}} \gamma \nabla S_{A}(.)\right\|_{L\left(\left(H^{1 / 2}\left(\Gamma^{*}\right)\right)^{n}, H^{1}(\bar{\Omega})\right)} \leq M_{\Gamma^{*}} e^{\alpha_{\Gamma^{*}}, \forall t \geq 0 .}
$$

Remark 2.2: If the semi-group $\left(S_{A}(t)\right)_{t \geq 0}$ is $\Gamma^{*} A G$-stable on $\left(H^{1 l 2}\left(\Gamma^{*}\right)\right)^{n}$, then for all $x_{o} \in H^{1}(\Omega)$, the solution of the associated system satisfies

$$
\begin{gathered}
\lim _{t \rightarrow \infty}\left\|\chi_{\Gamma^{*}} \gamma \nabla x(., t)\right\|_{\left(H^{1 / 2}\left(\Gamma^{*}\right)\right)^{n}}=\lim _{t \rightarrow \infty}\left\|\chi_{\Gamma^{*}} \gamma \nabla S_{A}(t) x_{0}\right\|_{\left(H^{1 / 2}\left(\Gamma^{*}\right)\right)^{n}} \\
=0
\end{gathered}
$$

Definition 2.3: The system (1) is said to be $\Gamma^{*} A G$-stable on $\Gamma^{*}$ if the operator $A$ generates a semi-group which is $\Gamma^{*}$ AG-stable on the space $\left(H^{1 / 2}\left(\Gamma^{*}\right)\right)^{n}$.

Definition 2.4: The system (1)-(2) is said to be regionally boundary asymptotically gradient detectable $\left(\Gamma^{*} A G-\right.$ detectable) on $\Gamma^{*}$, if there exists an operator $H_{\Gamma^{*} A G}: R^{q} \rightarrow\left(H^{1 / 2}\left(\Gamma^{*}\right)\right){ }^{n}$, such that the operator $\left(A-H_{\Gamma^{*} A G} C\right)$ generates a strongly continuous semi-group $\left(S_{H_{\Gamma^{*} A G}}(t)\right)_{t \geq 0}$, which is $\Gamma^{*} A G$-stable on $\left(H^{1 / 2}\left(\Gamma^{*}\right)\right)^{n}$.

Proposition 2.5: If the system (1)-(2) is $\Gamma^{*} G$-observable on $\Gamma^{*}$, then it is $\Gamma^{*} A G$-detectable on $\Gamma^{*}$. This results gives the following inequality: $\exists k_{\Gamma^{*} \mathrm{AG}}>0$, such that

$$
\left.\| \chi_{\Gamma^{*}}\right\rangle \nabla S_{A}(.) x\left\|_{\left(H^{1 / 2}\left(\Gamma^{*}\right)\right)^{n}} \leq k_{\Gamma^{*} A G}\right\| C S_{A}(.) x \|_{L^{2}(0, \infty, 0)},
$$

for all $z \in\left(H^{1 / 2}\left(\Gamma^{*}\right)\right)^{n}$.

Proof: We conclude the proof of this proposition is conclude from the results on observability considering $\chi_{\Gamma^{*}} \nabla K^{*}$. We have the following forms $[21,24]$

1. Imf $\subset$ Img.

2. There exists $k>0$, such that

$$
\left\|f^{*} x^{*}\right\|_{E^{*}} \leq k\left\|g^{*} x^{*}\right\|_{F^{*}} \text {, for all } x^{*} \in G^{*}
$$

From the right hand said of above inequality $k_{\Gamma^{*} \mathrm{AG}}\left\|g^{*} x^{*}\right\|_{F^{*}}$, there exists $M_{\Gamma^{*} \mathrm{AG}}, \omega_{\Gamma^{*} \mathrm{AG}}>0$ with $k_{\Gamma^{*} \mathrm{AG}}<M_{\Gamma^{*} \mathrm{AG}}$ such that

$$
k_{\Gamma^{*} \mathrm{AG}}\left\|g^{*} \mathrm{x}\right\|_{F^{*}} \leq M_{\Gamma^{*} \mathrm{AG}} e^{-\omega_{\Gamma^{*} \mathrm{AG}} t}\left\|x^{*}\right\|_{F^{*}}
$$


where $E, F$ and $G$ be a reflexive Banach spaces and $f \in L(E, G), g \in L(F, G)$. If we apply this result, considered

$$
E=G=\left(H^{1 / 2}\left(\Gamma^{*}\right)\right)^{n}, F=\mathcal{O}, f=I d_{\left(H^{1 / 2}\left(\Gamma^{*}\right)\right)^{n}}
$$

and

$$
g=S_{A}^{*}(.) \chi_{\Gamma}^{*} \gamma^{*} \nabla^{*} C^{*}
$$

where $S_{A}($.$) is a strongly continuous semi-group generates by A$, which is $\Gamma^{*} A G$-stable on $\Gamma^{*}$, then it is $\Gamma^{*} A G$ detectable on $\Gamma^{*}$. Thus, the notion of $\Gamma^{*} A G$-detectability is a weaker property than the $\Gamma^{*} G$-observability [25-26].

\section{Sensors and $\Gamma^{*}$ AGFO-Observer}

In this section we present the sufficient conditions which are guarantee the existence of regional boundary asymptotic gradient full order observer ( $\Gamma^{*}$ AGFO-Observer) on $\Gamma^{*}$ which allows to construct a $\Gamma^{*}$ AGFO-estimator on $\Gamma^{*}$ of the state $\chi_{\Gamma^{*}} \gamma \nabla T x(\xi, t)$.

\subsection{Definitions and characterizations}

Definition 3.1: Suppose there exists a dynamical system with state $z(., t) \in Z$ given by

$$
\begin{cases}\frac{\partial \hat{z}}{\partial t}(\xi, t)=A \hat{z}(\xi, t)+B u(t)+H_{\Gamma^{*} A G}(C x(\xi, t)-C \hat{z}(\xi, t)) & Q \\ \hat{z}(\xi, 0)=\hat{z}_{o}(\xi) & \bar{\Omega} \\ \hat{z}(\eta, t)=0 & \Sigma\end{cases}
$$

In this case the operator $F_{\Gamma^{*} A G}$ in general case [13] is given by $F_{\Gamma^{*} A G}=A-H_{\Gamma^{*} A G} C$ where $T=I$ the identity operator. Thus the operator $A-H_{\Gamma^{*} A G} C$ generate a strongly continuous semi-group $\left(S_{A-H_{\Gamma^{*} A G} C}(t)\right)_{t \geq 0}$ on separable Hilbert space $Z$ which is $\Gamma^{*} A G$-stable.

Thus, $\exists M_{A-H_{\Gamma^{*} A G} C}, \alpha_{A-H_{\Gamma^{*} A G} C}>0$ such that

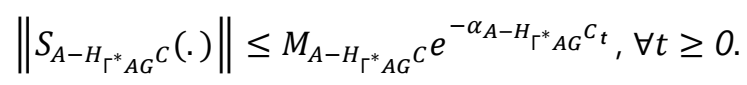

and let $G_{\Gamma^{*} A G} \in L(U, Z), H_{\Gamma^{*} A G} \in L(\mathcal{O}, Z)$ such that solution of (18) similar to (3)

$$
z(\xi, t)=S_{A-H_{\Gamma^{*} A G} C}(t) z(\xi)+\left[\int_{O}^{t} S_{A-H_{\Gamma^{*}}{ }_{A G} C}(t-\tau) B u(\tau) H_{\Gamma^{*} A G} y(\tau)\right] d \tau .
$$

Definition 3.2: The system (18) defines $\Gamma^{*} A G F O-e s t i m a t o r$ such that

$$
z(\xi, t)=\chi_{\Gamma} \nabla T x(\xi, t)=I x(\xi, t) \in\left(H^{1 / 2}\left(\Gamma^{*}\right)\right)^{n}
$$

where $x(\xi, t)$ is the solution of the systems (1)-(2) if

$$
\lim _{t \rightarrow \infty}\|z(., t)-x(\xi, t)\|_{\left(H^{1 / 2}\left(\Gamma^{*}\right)\right)^{n}}=0,
$$

and $\chi_{\ulcorner} \nabla \mathrm{I}$ maps $D(A)$ into $D\left(A-H_{\Gamma^{*} A G} C\right)$ where $z(\xi, t)$ is the solution of system (18).

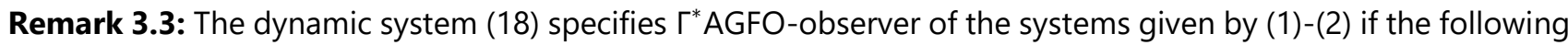
holds: 
1-There exists $M_{\Gamma^{*} \text { AGFO }} \in L\left(R,\left(H^{1 / 2}\left(\Gamma^{*}\right)\right)^{n}\right)$ and $N_{\Gamma^{*} \text { AGFO }} \in L\left(\left(H^{1 / 2}\left(\Gamma^{*}\right)\right)^{n}\right)$ such that

$$
\begin{aligned}
& i-M_{\Gamma^{*} \mathrm{AGFO}} C+N_{\Gamma^{*} \mathrm{AGFO}}=I_{\Gamma^{*} \mathrm{AGFO}} \\
& i i-A-F_{\Gamma^{*} \mathrm{AGFO}}=H_{\Gamma^{*} \mathrm{AGFO}} C \text { and } G_{\Gamma^{*} \mathrm{AGFO}}=B .
\end{aligned}
$$

3- The system (18) defines $\Gamma^{*}$ AGFO-estimator for $x(\xi, t)$.

The object of $\Gamma^{*}$ AGFO-observer is to provide an approximation to the original system state gradient. This approximation is given by

$$
\hat{x}(t)=M_{\Gamma^{*} \mathrm{AGFO}} y(t)+N_{\Gamma^{*} \mathrm{AGFO}} z(t) .
$$

Definition 3.4: The systems (1)-(2) are regionally boundary asymptotic gradient full order observable ( $\Gamma^{*} A G F O-$ observable) on $\Gamma^{*}$, if there exists a dynamic system which is $\Gamma^{*} A G F O-o b s e r v e r$ for the considered system.

\section{2 $\Gamma^{*}$ AGFO-Observer reconstruction method}

In this case, we need to consider $\chi_{\Gamma^{*} \mathrm{AGFO}} \nabla \mathrm{T}=I_{\Gamma^{*} \mathrm{AGFO}}$ and $Z=X$, then the operator observer equation becomes as $F_{\Gamma^{*} \mathrm{AGFO}}=A-H_{\Gamma^{*} \mathrm{AGFO}} C$ where $A$ and $C$ are known. Thus, the operator $H_{\Gamma^{*} \mathrm{AGFO}}$ must be determined such that the operator $F_{\Gamma^{*} \mathrm{AGFO}}$ is $\Gamma^{*} \mathrm{AG}$-stable. This observer is an extension of asymptotic observer as in [14-16, 24-31]. Now consider again system (1) together with output function (2) described by the following form

$$
\begin{cases}\frac{\partial x}{\partial t}=A x(\xi, t)+B u(t) & Q \\ x(\xi, 0)=x_{0}(\xi) & \bar{\Omega} \\ x(\eta, t)=0 & \Sigma \\ y(t)=C x(., t) & Q\end{cases}
$$

(19) Let $\Gamma^{*}$ be a given

sub-domain of $\bar{\Omega}$ and suppose that $\left.I_{\Gamma^{*} \text { AGFO }} \in \mathcal{L}\left(H^{1}(\Omega)\right)^{n}\right)$, and $\chi_{\Gamma^{*} \mathrm{AGFO}} \nabla T x(\xi, t)=\chi_{\Gamma^{*} \mathrm{AGFO}} \nabla x(\xi, t)$ there exists a system with state $z(\xi, t)$ such that

$$
z(\xi, t)=\chi_{\Gamma^{*} \mathrm{AGFO}} \nabla T x(\xi, t)=T_{\Gamma^{*} \mathrm{AGFO}} x(\xi, t)
$$

(with $T_{\Gamma^{*} \mathrm{AGFO}}=I_{\Gamma^{*} \mathrm{AGFO}}$ where $I_{\Gamma^{*} \mathrm{AG}}$ is the identity operator with respect to $\Gamma^{*} \mathrm{AGFO}$-estimator. Then

$$
z(\xi, t)=I_{\Gamma^{*} \mathrm{AGFO}} x(\xi, t)=x(\xi, t)
$$

From equation (2) and (21) we have

$$
\left[\begin{array}{l}
y \\
z
\end{array}\right]=\left[\begin{array}{c}
C \\
I_{\Gamma^{*} \mathrm{AGFO}}
\end{array}\right] x
$$

If we assume that there exist two bounded linear operators

$$
M_{\Gamma^{*} \mathrm{AGFO}}: \vartheta \rightarrow\left(\left(H^{1 / 2}\left(\Gamma^{*}\right)\right)^{n}\right.
$$

and

$$
N_{\Gamma^{*} \mathrm{AGFO}}:\left(( H ^ { 1 / 2 } ( \Gamma ^ { * } ) ) ^ { n } \rightarrow \left(\left(H^{1 / 2}\left(\Gamma^{*}\right)\right)^{n}\right.\right.
$$

such that

$$
M_{\Gamma^{*} \mathrm{AGFO}} C+N_{\Gamma^{*} \mathrm{AGFO}} T_{\Gamma^{*} \mathrm{AGFO}}=I_{\Gamma^{*} \mathrm{AGFO}}
$$

then by deriving $z(\xi, t)$ in (20) we have 


$$
\begin{aligned}
& \frac{\partial z}{\partial t}(\xi, t)=I_{\Gamma^{*} \mathrm{AGFO}} \frac{\partial x}{\partial t}(\xi, t)=\chi_{\Gamma^{*} \mathrm{AGFO}} \nabla T A x(\xi, t)+\chi_{\Gamma^{*} \mathrm{AGFO}} \nabla T B u(t) \\
& =\chi_{\Gamma^{*}} \nabla I_{\Gamma^{*} \mathrm{AGFO}} A M_{\Gamma^{*} \mathrm{AGFO}} y(\xi, t)+\chi_{\Gamma^{*}} \nabla I_{\Gamma^{*} \mathrm{AGFO}} A N_{\Gamma^{*} \mathrm{AGFO}} Z(\xi, t) \\
& +\chi_{\Gamma^{*}} \nabla I_{\Gamma^{*} \mathrm{AGFO}} B u(t)
\end{aligned}
$$

Since the operator $T_{\Gamma^{*} \mathrm{AGFO}}=I_{\Gamma^{*} \mathrm{AGFO}}$, then we have

$$
\frac{\partial z}{\partial t}(\xi, t)=A N_{\Gamma^{*} \mathrm{AGFO}} Z(\xi, t)+B u(t)+A M_{\Gamma^{*} \mathrm{AGFO}} y(\xi, t)
$$

Therefore

$$
\frac{\partial \hat{z}}{\partial t}(\xi, t)=F_{\Gamma^{*} G} \hat{z}(\xi, t)+G_{\Gamma^{*} G} u(t)+H_{\Gamma^{*} G} y(., t)
$$

and since $A-F_{\Gamma^{*} \mathrm{AGFO}}=H_{\Gamma^{*} \mathrm{AGFO}} C$ and $G_{\Gamma^{*} \mathrm{AGFO}}=B$ then we have

$$
\left\{\begin{array}{lr}
\frac{\partial \hat{z}}{\partial t}(\xi, t)=A \hat{z}(\xi, t)+B u(t)+H_{\Gamma^{*} \mathrm{AGFO}}(C x(\xi, t)-c C(\xi, t)) & Q \\
\hat{z}(\xi, 0)=\hat{z_{0}}(\xi) & \bar{\Omega} \\
\hat{z}(\eta, t)=0 & \bar{\Sigma}
\end{array}\right.
$$

Let us consider a complete sets of eigenfunctions $\varphi_{n j}$ in $\left(H^{1}(\Omega)\right)^{n}$ orthonormal to $\left(H^{1 / 2}\left(\Gamma^{*}\right)\right)^{n}$ associated with the eigenvalue $\lambda_{n}$ of multiplicity $r_{n}$ and suppose the system (1) has unstable mode. Then, the sufficient condition of an $\Gamma^{*} A G F O-o b s e r v e r$ is formulated in the following main result.

Theorem 3.5: Suppose that there are $q$ zone sensors $\left(D_{i}, f_{i}\right)_{1 \leq i \leq q}$ and the spectrum of $A$ contains $J$ eigenvalues with non-negative real parts. Then the dynamic system (22) is $\Gamma^{*} A G F O-o b s e r v e r ~ o n ~ \Gamma^{*}$ for the system (19), that is $\lim _{t \rightarrow \infty}[z(\xi, t)-\hat{z}(\xi, t)]=0$, if :

1-There exists

$M_{\Gamma^{*} \text { AGFO }} \in L\left(R^{q},\left(H^{1 / 2}\left(\Gamma^{*}\right)\right)^{n}\right)$ and $N_{\Gamma^{*} \text { AGFO }} \in L\left(\left(H^{1 / 2}\left(\Gamma^{*}\right)\right)^{n}\right)$ such that

$$
M_{\Gamma^{*} \mathrm{AGFO}} C+N_{\Gamma^{*} \mathrm{AGFO}}=I_{\Gamma^{*} \mathrm{AGFO}} .
$$

2- $A-F_{\Gamma^{*} \mathrm{AGFO}}=H_{\Gamma^{*} \mathrm{AGFO}} C, G_{\Gamma^{*} \mathrm{AGFO}}=B$.

3- $q \geq m$

4- rank $G_{m}=m_{m}, \forall m, m=1, \ldots, J$ with

$$
G_{m}=\left(G_{m}\right)_{i j}=\left\{\begin{array}{l}
\psi_{m j}\left(b_{i}\right), f_{i}(.)>_{L^{2}(D i)} \\
\psi_{m j}\left(b_{i}\right) \\
\left\langle\frac{\partial \psi_{m j}}{\partial v}, f i(.)>L^{2}\left(\Gamma^{*}{ }_{i}\right\rangle\right.
\end{array}\right.
$$

where $\sup m_{m}=m<\infty$ and $j=1, \ldots, m_{m}$.

\section{Proof:}

First step: The proof is limited to the case of pointwise sensors. Under the assumptions of section 2, the system (1) can be decomposed by the projections $P$ and $I-P$ on two parts, unstable and stable. The state vector may 
be given by $x(\xi, t)=\left[x_{1}(\xi, t), x_{2}(\xi, t)\right]^{\mathrm{t}}$ where $x_{1}(\xi, t)$ is the state component of the unstable part of the system (1) may be written in the form

$$
\left\{\begin{array}{lr}
\frac{\partial x_{1}}{\partial t}(\xi, t)=A_{1} x_{1}(\xi, t)+P B u(t) & Q \\
x_{1}(\xi, 0)=x_{01}(\xi) & \bar{\Omega} \\
x_{1}(\eta, t)=0 & \Theta
\end{array}\right.
$$

and $x_{2}(\xi, t)$ is the component state of the stable part of the system (1) given by

$$
\left\{\begin{array}{lr}
\frac{\partial x_{2}}{\partial t}(\xi, t)=A_{2} x_{2}(\xi, t)+(I-P) B u(t) & Q \\
x_{2}(\xi, 0)=x_{02}(\xi) & \bar{\Omega} \\
x_{2}(\eta, t)=0 & \Theta
\end{array}\right.
$$

The operator $A_{1}$ is represented by a matrix of order $\left(\sum_{m=1}^{J} m_{m}, \Sigma_{m=1}^{J} m_{m}\right)$ given by $A_{1}=\operatorname{diag}$ $\left[\lambda^{1}, \ldots, \lambda^{1}, \lambda^{2}, \ldots, \lambda^{2}, \ldots, \lambda_{J}, \ldots, \lambda_{J}\right]$ and $P B=\left[G_{1}{ }^{{ }^{r}}, G_{2}{ }^{{ }{ }}, \ldots, G_{J}{ }^{r}\right]$.The condition (4) of this theorem, allows that the suit $\left(D_{i}, f_{i}\right)_{1 \leq i \leq q}$ of sensors is $\Gamma^{*} \mathrm{G}$-strategic for the unstable part of the system (1), the subsystem (23) is W $\Gamma^{*} \mathrm{G}$ observable [11] and since it is finite dimensional, then it is $E \Gamma^{*} G$ observable [27]. Therefore it is asymptotically $\Gamma^{*} A G$-detectable, and hence there exists an operator $H_{\Gamma^{*} A G}^{7}$ such that $\left(A_{1}-H_{\Gamma^{*} A G}^{1} C\right)$ which is satisfied the following:

$\exists, M_{A 1-H_{\Gamma^{*} A G}^{1} C^{C}}, \alpha_{A 1-H_{\Gamma^{*} A G}^{1} C}>0$ such that

$$
\left\|e^{\left(A 1-H_{\Gamma^{*} A G}^{1} C\right) t}\right\|_{\left(H^{1}\left(\Gamma^{*}\right)\right)^{n}} \leq M_{A 1-H_{\Gamma^{*} A G}^{1} C} e^{-\alpha A 1-H_{\Gamma^{*} A G^{1}} c^{t}}
$$

and then we have

$$
\left\|x_{1}(., t)\right\|_{\left(H^{1}\left(\Gamma^{*}\right)\right)^{n}} \leq M_{A 1-H_{\Gamma^{*} A G}^{1} C} e^{-\alpha A 1-H_{\Gamma^{*} C}^{1}}\left\|P x_{0}(.)\right\|_{\left(H^{1}\left(\Gamma^{*}\right)\right)^{n}}
$$

Since the semi-group generated by the operator $A_{2}$ is stable on $\left(H^{1 / 2}\left(\Gamma^{*}\right)\right)^{n}$, then there exist $M_{A 2-H_{\Gamma^{*} A G}^{2} C^{\prime}}, \alpha_{A 2-H_{\Gamma^{*} A G}^{2} C}>0[5]$ such that

$$
\begin{aligned}
& \left\|x_{2}(., t)\right\|_{\left(H^{1}\left(\Gamma^{*}\right)\right)}{ }^{n} \leq M_{A 1-H_{\Gamma^{*} A G}^{1} C^{C}} e^{-\alpha A 2-H_{\Gamma^{*} A G}^{2} C(t)}\|(I-P)\|_{\left(H^{1 / 2}\left(\Gamma^{*}\right)\right)^{n}} \\
& +\int_{0}^{t} M_{M_{A 1-H_{\Gamma^{*} A G}^{1} G^{C}}}, e^{-\alpha A 2-H_{\Gamma^{*} A G}^{2} C^{(t)}} H_{\Gamma^{*} A G}^{2} \quad\left\|(I-P) x_{0}(.)\right\|_{\left(H^{1 / 2}\left(\Gamma^{*}\right)\right)^{n}}\|u(\tau)\| d \tau
\end{aligned}
$$

and therefor $x(\xi, t) \rightarrow 0$ when $t \rightarrow \infty$. Finally, the system (23) are asymptotically $\Gamma^{*} A G$-detectable.

Second step: From equation (21), we have $z(\xi, t)=x(\xi, t)$ with the observer error is given by the following form

$$
e(\xi, t)=z(\xi, t)-\hat{z}(\xi, t)
$$

where $\hat{z}(\xi, t)$ is a solution of the dynamic system (22). Derive the above equation, and by using equation (19) and condition 2 , we can get the following forms

$$
\begin{aligned}
& \frac{\partial \varepsilon}{\partial t}(\xi, t)=\frac{\partial z}{\partial t}(\xi, t)-\frac{\partial \hat{z}}{\partial t}(\xi, t)=\frac{\partial x}{\partial t}(\xi, t)-\frac{\partial \hat{z}}{\partial t}(\xi, t) \\
& \quad=A x(\xi, t)+B u(t)-F_{\Gamma^{*} \text { AGFO }} \hat{z}(\xi, t)
\end{aligned}
$$




$$
\begin{aligned}
& -G_{\Gamma^{*}} u(t)-H_{\Gamma^{*} \mathrm{AGFO}} C x(\xi, t) \\
& =A x(\xi, t)-\left(A-H_{\Gamma^{*} \mathrm{AGFO} G} C\right) \hat{z}(\xi, t)-H_{\Gamma^{*} \mathrm{AGFO}} C x(\xi, t) \\
& =\left(A-H_{\Gamma^{*} \mathrm{AGFO}} C\right)(z(\xi, t)-\hat{z}(\xi, t)) \\
& =\left(A-H_{\Gamma^{*} \mathrm{AGFO}} C\right) e(\xi, t)
\end{aligned}
$$

Thus, from the first part of this proof we obtain

$$
e(\xi, t)=\left(A-H_{\Gamma^{*} \mathrm{AGFO}} C\right) e(0, t)
$$

is asymptotically $\Gamma^{*} G$-stable with

$$
e(0, t)=z_{0}(\xi)-\hat{z_{0}}(\xi)
$$

Then we have

$$
\begin{array}{r}
\|e(\xi, t)\|_{\left.\left(H^{1 / 2}\left(\Gamma^{*}\right)\right)^{m}\right)} \leq M_{A-H_{\Gamma^{*} A G F O} C} e^{-\alpha A-H_{\Gamma^{*} A G F O} C^{t}} . \\
\left\|z_{0}(\xi)-\hat{z}_{0}(\xi)\right\|_{\left.\left(H^{1 / 2}\left(\Gamma^{*}\right)\right)^{m}\right)}
\end{array}
$$

therefore $\lim _{t \rightarrow \infty} e(\xi, t)$. Now, let the approximate solution to the gradient state of the original system is

$$
\hat{x}(\xi, t)=M_{\Gamma^{*} \mathrm{AGFO}} y(., t)+N_{\Gamma^{*} \mathrm{AGFO}} \hat{z}(\xi, t)
$$

with

$$
M_{\Gamma^{*} \mathrm{AGFO}}=0 \text { and } N_{\Gamma^{*} \mathrm{AGFO}}=I_{\Gamma^{*} \mathrm{AGFO}} \text {, }
$$

then we have

$$
\hat{x}(\xi, t)=\hat{z}(\xi, t)
$$

Now, we can calculate the error of gradient state estimator

$$
\begin{aligned}
\hat{e}_{\Gamma^{*} \mathrm{AGFO}}(\xi, t)= & x(\xi, t)-\hat{x}(\xi, t)=x(\xi, t)-x(\xi, t)+x(\xi, t)-\hat{x}(\xi, t) \\
& =\hat{x}(\xi, t)-\hat{z}(\xi, t)=e(\xi, t)=\left(A-H_{\Gamma^{*} \mathrm{AGFO}} C\right) e(0, t)
\end{aligned}
$$

is asymptotically $\Gamma^{*} G$-stable with

$$
e(0, t)=z_{0}(\xi)-\hat{z_{0}}(\xi) .
$$

Consequently, we get

$$
\lim _{t \rightarrow \infty}\|x(., t)-\hat{x}(\xi, t)\|_{\left(H^{1 / 2}\left(\Gamma^{*}\right)\right)^{n}}=\lim _{t \rightarrow \infty}\|x(., t)-\hat{z}(\xi, t)\|_{\left(H^{1 / 2}\left(\Gamma^{*}\right)\right)^{n}}=0
$$

Then, the dynamical system (22) is $\Gamma^{*} A G F O$-Observer to the system (19).

Corollary 3.6 From the previous results, we can deduce that:

1. Theorem 3.5 gives the sufficient conditions which guarantee the dynamic system (22) is a $\Gamma^{*} G F O$-observer for the system (19). 
2. If a system which is an $\Omega G F O$-observer, then it is $\Gamma^{*} A G F O$-observer for system (19).

3. If a system is $\Gamma^{*} G F O$-observer, then it is $\Gamma^{1 *} G F O$-observer for every subset $\Gamma^{1 *}$ of $\Gamma^{*}$, but the converse is not true [28]. This is an important case because there are many problems in real world can reconstruction the current state gradient but is not in the usual sense which is presented in the following example.

Example 3.7. Consider a two-dimensional system described by the diffusion equation

$$
\begin{cases}\frac{\partial x}{\partial t}\left(\xi_{1}, \xi_{2}, t\right)=\Delta x\left(\xi_{1}, \xi_{2}, t\right) & \mathcal{Q} \\ x\left(\xi_{1}, \xi_{2}, t\right)=x_{0}\left(\xi_{1}, \xi_{2}\right) & \bar{\Omega} \\ \frac{\partial x}{\partial v}\left(\eta_{1}, \eta_{1}, t\right)=0 & \Theta\end{cases}
$$

where $\Omega=] 0,1[\times] 0,1\left[\right.$. The operator $A=\Delta$ generates a strongly continuous semi-group $\left(S_{A}(t)\right)_{t \geq 0}$ on the Hilbert space $H^{1}(\Omega)$ given by

$$
S_{A}(t) x=\sum_{n, m=0}^{\infty} e^{\lambda_{n m}} t\left\langle x, \varphi_{n m}\right\rangle_{H^{1}(\Omega)} \varphi_{n m}
$$

With

$$
\begin{aligned}
& \lambda_{n m}=-\left(n^{2}, m^{2}\right) \pi^{2}, \varphi_{n m}\left(\xi_{1}, \xi_{2}\right)=2 a_{n m} \cos \left(n \pi \xi_{1}\right) \cos \left(n \pi \xi_{2}\right) \text { and } \\
& 2 a_{n m}=\left(1-\lambda_{n m}\right)^{-1 / 2} .
\end{aligned}
$$

Consider the dynamical system

$$
\left\{\begin{array}{lr}
\frac{\partial x}{\partial t}\left(\xi_{1}, \xi_{2}, t\right)=\Delta x\left(\xi_{1}, \xi_{2}, t\right)-H_{A G F O} C\left(x\left(\xi_{1}, \xi_{2}, t\right)-z\left(\xi_{1}, \xi_{2}, t\right)\right) & \mathcal{Q} \\
x\left(\xi_{1}, \xi_{2}, 0\right)=x_{0}\left(\xi_{1}, \xi_{2}\right) & \bar{\Omega} \\
\frac{\partial x}{\partial v}\left(\eta_{1}, \eta_{1}, t\right)=0 & \Theta
\end{array}\right.
$$

Where $H \in L\left(R^{q}, Z\right), Z$ is a Hilbert space and $C: H^{1}(\bar{\Omega}) \rightarrow R^{q}$ is a linear operator. Consider the boundary sensor $\left(\Gamma_{0}, f\right)$ defined by

$$
\left.\Gamma_{0}=\{0\} \times\right] 0,1\left[\text { and } f\left(\eta_{1}, \eta_{2}\right)=\cos \pi \eta_{2}\right.
$$

Thus, the output function can be written by

$$
y(t)=\int_{\Gamma_{0}} x\left(\eta_{1}, \eta_{2}, t\right) f\left(\eta_{1}, \eta_{2}\right) d \eta_{1} d \eta_{2}
$$

If the state $x_{0}$ is defined in $\Omega$ by

$$
x_{0}\left(\xi_{1}, \xi_{2}\right)=\cos \left(\pi \xi_{1}\right) \cos \left(2 \pi \xi_{2}\right),
$$

then the system (20)-(22) is not W $\Omega$ G-observable, i.e. the sensor $\left(\Gamma_{0}, f\right)$ is not $\Omega$-strategic and therefore the system (20)-(22) is not $\Omega A G$-detectable [7]. Thus, the dynamical system (21) is not $\Omega A G$-observer for the system (20)-(22) (see [29]). Here, we consider the region $\Gamma=] 0,1[\times\{0\} \subset \partial \Omega$ (figure 3 ) and the dynamical system 


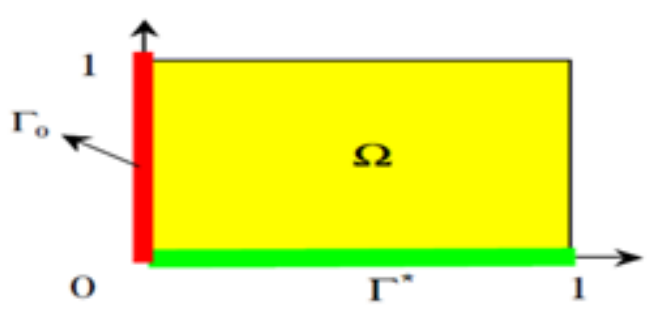

Fig. 3: Domain $\Omega$, region $\Gamma^{*}$ and location $\Gamma_{0}$ of zone sensor.

$$
\left\{\begin{aligned}
\frac{\partial x}{\partial t}\left(\xi_{1}, \xi_{2}, t\right) & =\Delta x\left(\xi_{1}, \xi_{2}, t\right) & & \\
& -H_{\Gamma^{*} \mathrm{AGFO \Gamma} C} C\left(x\left(\xi_{1}, \xi_{2}, t\right)-z\left(\xi_{1}, \xi_{2}, t\right)\right) & & \mathcal{Q} \\
x\left(\xi_{1}, \xi_{2}, 0\right) & =x_{0}\left(\xi_{1}, \xi_{2}\right) & & \bar{\Omega} \\
\frac{\partial x}{\partial v}\left(\eta_{1}, \eta_{1}, t\right) & =0 & & \Theta
\end{aligned}\right.
$$

where $H \in L\left(R^{q}, H^{1 / 2}\left(\Gamma^{*}\right)\right)$. In this case, the system (20)-(22) is W $\Gamma^{*} G$-observable and the sensor $\left(\Gamma_{0}, f\right)$ is $\Gamma^{*} G$ strategic [26]. Thus, the system (20)-(22) is $\Gamma^{*} A G$-detectable [13]. Finally the dynamical system (20) is $\Gamma^{*} A G F O$ observer for the system (20)-(22) [30].

\section{Application to asymptotic $\Gamma^{*} A G F O$-observer in diffusion system}

In this section we consider the distributed diffusion systems defined in the domain $\Omega$. Various results related to different types of sensor have been extended. In the case of two-dimensional, we take where $\Omega=] 0, a_{1}[\times] 0, a_{2}[$ and $\left.\Gamma^{*}=\right] 0, a_{1}\left[\times\left\{a_{2}\right\}\right.$ is a region of $\partial \Omega$ the boundary of $\Omega$. The eigenfunctions of the dynamic system (16) for Dirichlet boundary conditions are given by

$$
\varphi_{n m}\left(\mu_{1}, \mu_{2}\right)=\left(\frac{4}{a_{1} a_{2}}\right)^{1 / 2} \cos n \pi\left(\frac{\mu_{1}}{a_{1}}\right) \cos m \pi\left(\frac{\mu_{2}}{a_{2}}\right)
$$

associated with eigenvalues

$$
\lambda_{n m}=-\left(\frac{n^{2}}{a_{1}^{2}}+\frac{m^{2}}{a_{2}^{2}}\right) \pi^{2}, n, m \geq 1
$$

If we suppose that $a_{1}^{2} / a_{2}^{2} \notin Q$, then the multiplicity of the eigenvalues $\lambda_{n m}$ is $r_{n m}=1$ for every $n, m=\{1, \ldots, J\}$, then one sensor $(D, f)$ may be sufficient for $\Gamma^{*} A G F O$-observer [24].

\subsection{Zone case in rectangular domain}

This subsection related to give sufficient conditions which is characterized some cases of the $\Gamma^{*} A G F O$-observer in the rectangular domain of system (12) with various sensor locations cases.

\subsubsection{Internal zone sensors case}

Consider two dimensional system defined in $\bar{\Omega}=\left[0, a_{7}\right] \times\left[0, a_{2}\right]$ by parabolic equation

$$
\begin{cases}\frac{\partial x}{\partial t}\left(\xi_{1}, \xi_{2}, t\right)=\frac{\partial^{2} x}{\partial \xi_{1}^{2}}\left(\xi_{1}, \xi_{2}, t\right)+\frac{\partial^{2} x}{\partial \xi_{2}^{2}}\left(\xi_{1}, \xi_{2}, t\right) & Q \\ x\left(\xi_{1}, \xi_{2}, 0\right)=x_{0}\left(\xi_{1}, \xi_{2}\right) & \bar{\Omega} \\ x\left(\eta_{1}, \eta_{2}, t\right)=0 & \Sigma\end{cases}
$$

augmented with output function measured by internal or boundary zone sensors 


$$
y(., t)=\int_{D} x\left(\xi_{1}, \xi_{2}, t\right) f\left(\xi_{1}, \xi_{2}\right) d \xi_{1} d \xi_{2}
$$

where the zone sensor is the located inside the domain $\Omega$, over the support

$$
D=] \xi_{1_{0}}-l_{1}, \xi_{1_{0}}+l_{1}[\times] \xi_{2_{0}}-l_{2}, \xi_{2_{0}}+l_{2}\left[\subset \Omega \text {, and } f \in L^{2}(D)\right.
$$

as in (figure 4).

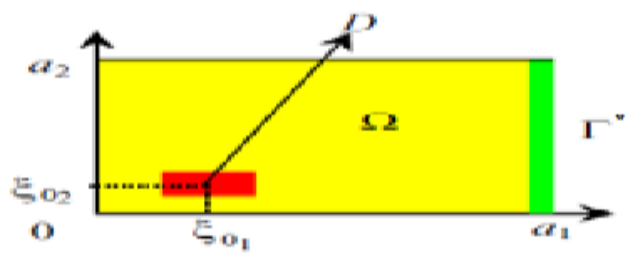

Fig. 4: Rectangular domain $\Omega$, region $\Gamma^{*}$ and location $D$ of internal zone sensor.

In this case the system (31)-(32) have an associated dynamical system is given by the following form

$$
\begin{cases}\frac{\partial z}{\partial t}\left(\xi_{1}, \xi_{2}, t\right)=\frac{\partial^{2} z}{\partial \xi_{1}^{2}}\left(\xi_{1}, \xi_{2}, t\right)+\frac{\partial^{2} z}{\partial \xi_{2}^{2}}\left(\xi_{1}, \xi_{2}, t\right) & \\ -H_{\Gamma^{*} G}\left(C z\left(\xi_{1}, \xi_{2}, t\right)-y(t)\right) & Q \\ z\left(\xi_{1}, \xi_{2}, 0\right)=z_{0}\left(\xi_{1}, \xi_{2}\right) & \bar{\Omega} \\ z\left(\eta_{1}, \eta_{2}, t\right)=0 & \Sigma\end{cases}
$$

Thus have the following important result.

Proposition 4.1: Suppose that $f_{1}$ is symmetric about $\xi=\xi_{01}$ and $f_{2}$ is symmetric about $\xi=\xi_{02}$, then the dynamical system (33) is $\Gamma^{*} A G F O$-observer for systems (31)-(32) if $n \mu_{1_{0}} / a_{1}$ and $m \mu_{2_{0}} / a_{2} \notin N$, for every $n, m=$ $\{1, \ldots, J\}$.

\subsubsection{One side boundary zone sensor case}

Now, the measurements are given by the following output function

$$
y(t)=\int_{\Gamma_{0}} \frac{\partial x}{\partial v}\left(\eta_{1}, \eta_{2}, t\right) f\left(\eta_{1}, \eta_{2}\right) d \eta_{1} \eta_{2}
$$

Where $\Gamma_{0} \subset \partial \Omega$ is the boundary support of the sensor and $f \in L^{2}\left(\Gamma_{0}\right)$. In the case, where the support of the sensor $\left(\Gamma_{0}, f\right)$ is one of side as in (figure 5$)$

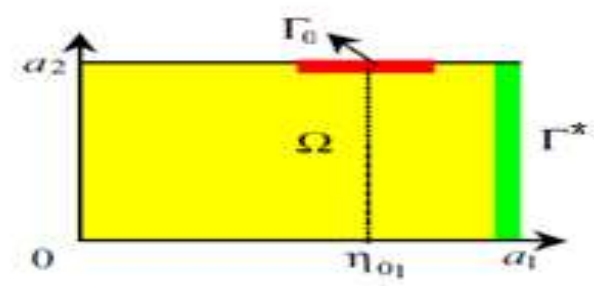

Fig. 5: Rectangular domain $\Omega$, region $\Gamma^{*}$ and location $\Gamma_{0}$ of boundary zone sensor.

Then, we have the following result. 
Proposition 4.2: Suppose that $\Gamma^{*} \subset \partial \Omega$ and $f$ is symmetric with respect to $\eta_{1}=\eta_{0_{1}}$, then the dynamic system (33) is $\Gamma^{*} A G F O$-observer for the systems (31)-(32) if $n \eta_{1_{0}} / a_{1} \notin N$, for all $n, n=\{1, \ldots, J\}$.

\subsubsection{Two side boundary zone sensor case}

In this case the output function (2) is given by

$$
y(t)=\int_{\bar{\Gamma}} \frac{\partial x}{\partial v}\left(\eta_{1}, \eta_{2}, t\right) f\left(\eta_{1}, \eta_{2}\right) d \eta_{1} \eta_{2}
$$

when the

support of the sensor is on two sides boundary, i.e.,

$$
\bar{\Gamma}=\left[0, \bar{\eta}_{1_{0}}+l_{7}\right] \times\{0\} \cup\{0\} \times\left[0, \bar{\eta}_{2_{0}}+l_{2}\right]=\bar{\Gamma}_{1} \cup \bar{\Gamma}_{2} \subset \partial \Omega,
$$

as in (figure 6),

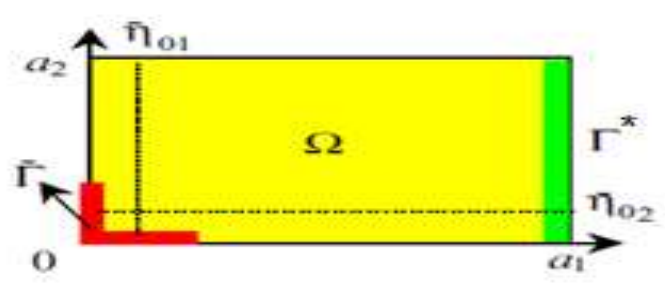

Fig. 6: Rectangular domain $\Omega$, region $\Gamma^{*}$ and location $\bar{\Gamma}^{*}$ of boundary zone sensor.

then, we obtain the following result.

Proposition 4.3: Let $\bar{\Gamma}_{1} \cup \bar{\Gamma}_{2} \subset \partial \Omega$, the function $\left.f\right|_{\Gamma_{1}}$ is symmetric with respect to $\eta_{1}=\bar{\eta}_{01}$, and the function $\left.f\right|_{\Gamma_{2}}$ is symmetric with respect to $\eta_{2}=\bar{\eta}_{02^{\prime}}$ then the dynamic system (33) is $\Gamma^{*} A G F O$-observer for the systems (31)(32) if $n \eta_{1_{0}} / a_{1} \notin N$, for all $n, n=\{1, \ldots, J\}$.

\subsubsection{Pointwise sensors case}

Consider again the systems (31)-(2) augmented with output function measured by internal pointwise sensors. In this case, the output function is given by the following form

$$
y(t)=x\left(\xi_{1}, \xi_{2}, t\right) \delta\left(\xi_{1}-b_{1}, \xi_{2}-b_{2}\right) d \xi_{1} d \xi_{2}
$$

where $b=\left(b_{1}, b_{2}\right)$ is the location of pointwise sensor in $\Omega$ as defined in (figure 7).

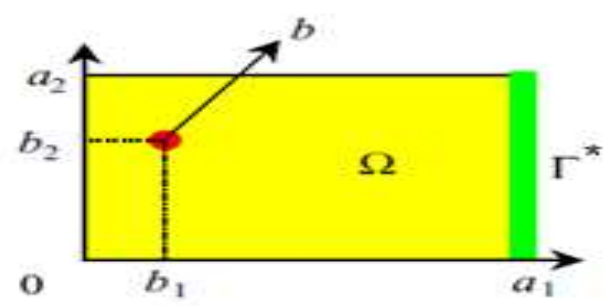

Fig. 7: Rectangular domain $\Omega$, region $\Gamma^{*}$ and location $b$ of internal pointwise sensors.

Then we obtain the following result.

Proposition 4.4: Let the sensor is located in $b=\left(b_{1}, b_{2}\right)$, then, the dynamic system (33) is $\Gamma^{*} A G F O$-observer for the systems (31)-(36), if $n b_{1} / a_{1}$ and $m b_{2} / a_{2} \notin N$, for every $n, m=\{1, \ldots, J\}$. 


\subsubsection{Filament pointwise case}

Suppose that the observation is given by the filament sensor where $\sigma=\operatorname{Im}(\gamma) \subset \Omega$ is symmetric with respect to the line $b=\left(b_{1}, b_{2}\right)$ as in (figure 8). More precisely, the sensor is line of pointwises inside the domain $\Omega$, then the output function still given by equation (31

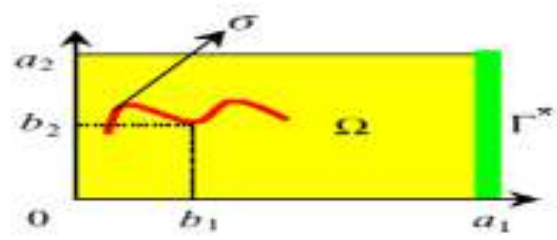

Fig. 8: Rectangular domain, region $\Gamma^{*}$ and locations $\sigma$ of filament pointwise sensor.

Proposition 4.5: Let the sensor is located in $b=\left(b_{1}, b_{2}\right)$, then, the dynamic system (33) is $\Gamma^{*} A G F O$-observer for the systems (31)-(36), if $n b_{1} / a_{1}$ and $m b_{2} / a_{2} \notin N$, for every $n, m=\{1, \ldots, J\}$.

\subsubsection{Boundary pointwise case}

Suppose that the sensor $\left(b, \delta_{b}\right)$ is located on $b$, where $b=\left(b_{1}, b_{2}\right) \in \partial \Omega$ with $b=\left(0, b_{2}\right)$ as in (figure 9).

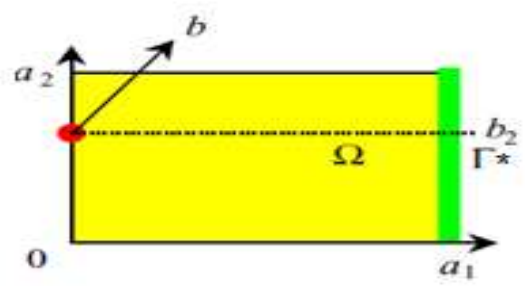

Fig. 9: Rectangular domain, region $\Gamma^{*}$ and location $b$ of boundary pointwise sensor.

In this case, the output function is given by

$$
\begin{aligned}
y(., t)= & \int_{D} x\left(\eta_{1}, \eta_{2}, t\right) \delta_{b}\left(\eta_{1}, \eta_{2}-b_{2}\right) d \eta_{1} \eta_{2}(y(., t) \\
& =\int_{\partial \Omega} \frac{\partial x}{\partial v}\left(\eta_{1}, \eta_{2}, t\right) f\left(\eta_{1}, \eta_{2}\right) d \eta_{1} d \eta_{2}
\end{aligned}
$$

where $b=\left(0, b_{2}\right)$. Thus, we obtain the following result.

Proposition 4.6: Let the sensor is located in $b=\left(0, b_{2}\right)$, then, the dynamic system (33) is $\Gamma^{*} A G F O$-observer for the systems (31)-(37), if $m b_{2} / a_{2} \notin N$ for all $m=\{1, \ldots, J\}$.

Now, from the previous results in this paper, we can deduce the following results.

Remark 4.7: We can extend these results to the case of two dimensional systems with circular domain in different sensor structures as in $[13,16]$.

Remark 4.8: We can extend the above results of the two dimensional systems (31)-(2) to case of one dimensional systems case with $\Omega=] 0, a$ [ as in ref.s $[8-17,25]$.

Remark 4.9: We know that the previous results have been developed with Dirichlet boundary conditions, then we can extend with Neumann or mixed boundary conditions as in [10, 31]. 


\section{Conclusion}

The concept have been studied in this paper is related to the $\Gamma^{*} A G F O$-observer in connection with sensors structure for a class of distributed parameter systems. More precisely, we have been given a sufficient condition for the existing an $\Gamma^{*} A G F O$-observer which allows to estimate the gradient state in a subregion $\Gamma^{*}$. For future work, one can be extension these result to the problem of regional boundary asymptotic gradient reduced order observer in connection with the sensors structures.

Acknowledgments. Our thanks in advance to the editors and experts for their efforts.

\section{References}

1. B. Friedland, Full-order state observer, Control Systems, Robotic and Automation, 10 (1996). https://www.eolss.net/Sample-Chapters/C18/E6-43-13-08.pdf

2. R. Al-Saphory, Strategic sensors and regional exponential observability, ISRN Applied Mathematics, (2011), 1-13. https://www.hindawi.com/journals/isrn/2011/673052/

3. R. Al-Saphory and Sh. Al-Mullah, Regional Exponential Reduced Observability in Distributed Parameter Systems, Journal of Advance in Mathematics, 11 (2015), 5058-5074.

https://raipub.com/index.php/iam/article/view/1256

4. R. Al-Saphory and Z. Jaafar, Regional exponential general observability in distributed parameter systems, Journal of Advance in Mathematics, 11 (2015), 5166-5182.

https://www.scribd.com/document/283351193/REGIONAL-EXPONENTIAL-GENERAL-OBSERVABILITYIN-DISTRIBUTED-PARAMETER-SYSTEMS

5. D. G. Luenberger, Observers For multivariable systems, IEEE Trans. Automat. Contr., 11 (2) (1966), 190197. https://ieeexplore.ieee.org/document/1098323

6. R.V. Gressang and G.B. Lamont, Observers for characterized by semi-groups, IEEE Trans. Automat. Contr., 20 (1975), 523-528. https://ieeexplore.ieee.org/document/1101021

7. A. El Jai and M. Amouroux, Sensors and observers in distributed parameter systems, International Journal of Control., 47 (1988), 333-347.

https://www.tandfonline.com/doi/abs/10.1080/00207178808906013

8. R. Al-Saphory, M. Al-Joubory and M. Jasim, Regional strategic sensors characterizations, Journal Mathematical and Computational Science, 3 (2013), 401-418.

http://scik.org/index.php/imcs/article/view/810

9. A. El Jai and H. Hamzauoi, Regional observation and sensors, International Journal of Applied Mathematics and Computer Sciences, 19 (2009), 5-14.

https://www.sciencedirect.com/science/article/abs/pii/092442479380204T

10. A. El Jai, M. C. Simon and E. Zerrik, Regional observability and sensor structure, Sensors and Actuators A: Physical, 39 (1993), 95-102. 


\section{https://www.sciencedirect.com/science/article/abs/pii/092442479380204T}

11. R. Al-Saphory, N. Al-Jawari and A. Al-Janabi, Regional gradient strategic sensors characterization, Journal of Advanced in Mathematics, 10 (2015), 3605-3618.

https://rajpub.com/index.php/jam/article/view/1735

12. E. Zerrik and H. Bourray, Gradient observability for diffusion systems, International Journal of Applied Mathematics and Computer Sciences, 2 (2003), 139-150.

http://pldml.icm.edu.pl/pldml/element/bwmeta1.element.bwnjournal-article-amcv13i2p139bwm

13. R. Al-Saphory, Asymptotic regional analysis for a class of distributed parameter systems, Ph.D. thesis, University of Perpignan, France, 2001, published in French, Amazon Google books, 2011.

https://books.google.iq/books/about/Analyse r\%C3\%A9gionale asymptotique d une cl.html?id=Trwj OgAACAAJ\&redir esc $=\mathbf{y}$

14. R. Al-Saphory and A. El Jai, Regional Asymptotic State Reconstruction, International Journal of System Science, 33 (2002), 1025-1037.

https://www.tandfonline.com/doi/abs/10.1080/00207720210166998

15. R. Al-Saphory, N. Al-Jawari and A. Al-Janabi, General asymptotic Regional gradient observer, Aust. J. Basic and Appl. Sci., 10 (2016), 8-18.

http://www.ajbasweb.com/old/ajbas May 2016.html

16. R. Al-Saphory and A. El-Jai, Sensors and asymptotic $\omega$-observer for distributed diffusion system, Sensors, 1 (2001), 161-182. https://www.mdpi.com/1424-8220/1/5/161/htm

17. J. Borggaard, J. Burns, A. Surana and L. Zietsman, Control, estimation and optimization of energy efficient buildings, Proc. of the American Control Conference, Hyatt Regency Riverfront, St. Louis, (2009), 837-841. https://ieeexplore.ieee.org/document/5160552

18. R Al-Saphory and M Al-Bayati, Regional boundary gradient detectability in distributed parameter systems, Journal of Advances in Mathematics, 14 (2), (2018), 7818-7833.

https://rajpub.com/index.php/jam/article/view/7478

19. A. El Jai, Distributed systems analysis via sensors and actuators. Sensors and Actuators A, 29,(1), (1991), $1-11$.

https://www.sciencedirect.com/science/article/abs/pii/092442479180026L

20. R. Curtain, and |H. Zwart, An introduction to infinite dimensional linear system theory, Springer-Verlag, New York, 1995. https://www.springer.com/gp/book/9780387944753

21. R. F. Curtain, and A. J. Pritchard, Infinite dimensional linear systems theory, Lecture Note in Control and Information Sciences, Springer- Verlag, New York, 1978.

https://www.springer.com/gp/book/9783540089612 
22. Burns, J., Observers and ooptimal estimation for PDE ssystems: sensor location problems, IMA Workshop on Sensor Location in Distributed Parameter Systems, September 6-8, 2017 Minneapolis, MN, USA. 2017. https://www.ima.umn.edu/2017-2018/SW9.6-8.17/26444

23. Brezis H., Analyse Fonctionnalle, Theorie et applications, 2em tirage, Masson, Paris, France,1987. https://www.dunod.com/sciences-techniques/analyse-fonctionnelle-theorie-et-applications

24. A. El Jai and A.J. Pritchard, Sensors and Controls in the Analysis of Distributed Parameter Systems, Ellis Hardwood Series in Mathematics and Applications, Wiley, New York, 1988.

https://www.ebay.co.uk/itm/Sensors-and-Controls-in-the-Analysis-of-Distributed-SystemsMathematics-and-its/153079971972?epid=91726288\&hash=item23a4470084:i:153079971972

25. R. Al-Saphory and A. El-Jai, Sensors structures and regional detectability of parabolic distributed system, Sensors and Actuators A: Physical, 90, (2001), 163-171.

https://www.sciencedirect.com/science/article/abs/pii/S0924424701005234

26. Al-Saphory, R. and El Jai, A., Sensors characterizations for regional boundary detectability in distributed parameter systems, Sensors and Actuators A, 94( 1-2), 2001, 1-10.

https://www.sciencedirect.com/science/article/abs/pii/S0924424701006690

27. R. Al-Saphory, N. Al-Jawari and A. Al-Janabi, Regional boundary gradient strategic sensors characterizations, Mathematical Theory and Modeling, 6 (2016), 10-26.

https://www.iiste.org/Journals/index.php/MTM/article/view/28228

28. El Jai, A.; Pritchard, A. J. Sensors and actuators in distributed parameter systems, International Journal of Control., 46, 1987, 1139-1153.

https://www.tandfonline.com/doi/abs/10.1080/00207178708933956?journalCode=tcon20

29. E. Zerrik, L. Badraoui, A. El Jai, Sensors and regional boundary state reconstruction of parabolic systems. Sensors and Actuators A, 75 (1999), 102-117.

http s://www.sciencedirect.com/science/article/abs/pii/S0924424798002933

30. R. Al-Saphory, Strategic sensors and regional exponential observability. ISRN Applied Mathematics, 2011. Article ID 673052, 1-13, 2011.

https://www.hindawi.com/journals/isrn/2011/673052/

31. R. Al-Saphory, Strategic sensors and regional observation in Neumann boundary conditions, Zanco Journal of Pure and Applied Science, Salahaddin University-Hawler/Iraq, 2010.

https://www.academia.edu/35033214/Regional Asymptotic Approach for Dynamical Free Systems 